\section{Charge Equalization for an Electric Vehicle Battery System}

NASSER H. KUTKUT, Member, IEEE

H. L. N. WIEGMAN

DEEPAK M. DIVAN, Senior Member, IEEE

D. W. NOVOTNY, Fellow, IEEE

University of Wisconsin-Madison

Charge equalization for series connected battery strings has important ramifications on battery life. It enhances the uniformity of the battery cells and hence improves the life of the battery as a whole. A new charge equalization technique for a series string of battery cells has been recently proposed by the authors. The basic technique utilizes a simple isolated dc-to-dc converter with a capacitive output filter along with a multiwinding transformer. The possibility of integrating the trickle charge function with the charge equalization function is potentially very attractive, as it can lead to an efficient and low cost implementation.

Manuscript received May 28, 1996; revised November 25, 1996.

IEEE Log No. T-AES/34/1/00192.

Authors' current addresses: N. H. Kutkut, Soft Switching Technologies, 2224 Evergreen Rd., Suite 6, Middleton, WI 53562; H. L. N. Wiegman, University of Wisconsin-Madison; D. M. Divan, Soft Switching Technologies, Middleton, WI (on leave from the University of Wisconsin-Madison); D. W. Novotny, Dept. of Electrical and Computer Engineering, University of Wisconsin-Madison, 1415 Johnson Dr., Madison, WI 53706.

$0018-9251 / 98 / \$ 10.00$ (C) 1998 IEEE

\section{INTRODUCTION}

Battery life is one of the major factors presently limiting the realization of economically viable electric vehicles (EVs). In an EV application, the main motive power source is realized using series connected battery strings with bus voltages in the range of 300-400 V. It has been shown that battery life, under a normal operating cycle, tends to degrade almost exponentially as the battery string length is increased. Series connected battery strings are prone to dramatic reduction in life and potential damage if high rate charging is continued after the onset of gassing. In order to improve battery life, individual cells need to be maintained at an equalized charge level.

A new technique for charge equalization of a series connected battery string has been proposed by the authors in [1]. The proposed technique utilizes the property of individual module voltages having the same value once they have reached the final state of charge. As a result, it is possible to use only one power converter to provide charge equalization for all the modules in the stack. In addition, the trickle charge function can be integrated with the charge equalizer to yield an efficient and low cost implementation. However, the main challenge in implementing such a technique is the ability to realize converters and magnetic components with tightly controlled parasitics and highly symmetrical transfer characteristics. The coaxial winding transformer (CWT) has been demonstrated to have these properties.

In this work, the design considerations for the proposed technique as applied to an EV battery system are discussed. Experimental data is provided to verify the concepts presented.

\section{PERFORMANCE OF LEAD ACID BATTERIES}

A lead acid battery cell consists of a positive electrode, a negative electrode, a porous separator between the two electrodes, and an electrolyte environment where all of these elements rest. Under normal operation, the electrodes undergo an oxidation-reduction (redox) reaction which affects the transfer of ions between them. The flow of ions is manifested as a charge flow that appears as battery current.

In general, the charging period of a lead acid battery can be divided into an efficient stage, a mixed stage, and a gassing stage. During the efficient stage, the predominant reactions are the transformation of $\mathrm{PbSO}_{4}$ into $\mathrm{Pb}$ and $\mathrm{SO}_{2}$. The charge acceptance, defined as the ratio of the current transformed into electrochemical storage to the current from the charger, is nearly $100 \%$. This stage is normally over when the state of charge reaches $70-80 \%$. During the mixed stage, water electrolysis processes proceed 
simultaneously with the main reactions. The charge acceptance is gradually and continuously reduced. Once the cell voltage shows no further increase, the battery cells are considered fully charged. In the gassing stage, the cells are fully charged while water decomposition and self discharge processes proceed in the battery. In fact, water electrolysis causes a rather large part of the energy loss in lead acid batteries. When a battery module is being charged, the level of current flowing into the module is controlled by the battery charging system. A high charging current level may well result in exceeding the natural redox reaction capacity of the cell electrodes. This would result in an electrolysis of the aqueous electrolyte away from the electrodes which frees gaseous oxygen and hydrogen instead of soluble oxygen and hydrogen ions. This will result in gas bubbles close to the electrode surfaces which block the flow of ions to those surfaces. Hence, the effective electrode surface area is reduced and the current handling capability is diminished. In addition, the presence of gas can result in pressure build up beyond the seal capability and possible leakage of hydrogen and oxygen gasses. Eventually, fluid loss will decrease the current handling capability of the battery cell.

The onset of overcharge can be detected by monitoring the battery voltage. Fig. 1 shows cell voltage versus capacity returned, as a percentage of previous charge, for different charge rates where $C$ is the amp-hour capacity of the battery cell [2]. The sharp rise in voltage indicates overcharge reactions. For the onset of overcharge reactions to coincide with $100 \%$ return of capacity, the charge rate must typically be less than $C / 100$.

\section{SERIES CONNECTED BATTERY MODULES}

As pointed out earlier, in an EV application the main power source is realized using series connected battery strings. Hence, individual battery modules are charged serially.

Different schemes and algorithms have been developed to achieve this task. One algorithm uses multistep constant current charging [3]. The charging process starts with the maximum current the charger can deliver to the battery pack at low state of charge. As the state of charge builds up, the charging current is reduced in steps. Rest (cooling) periods are also incorporated in the algorithm to minimize temperature differences between the cold and warm cells. In addition, equalization charging is applied at low current levels to improve the battery capacity.

A new fast-charging algorithm has been recently proposed by Podrazhansky [4]. It consists of three operational modes: an active-charge mode, an active-discharge mode and an inactive rest mode. During the first mode, positive pulses apply energy to the battery. In the second mode, a sharp depolarization

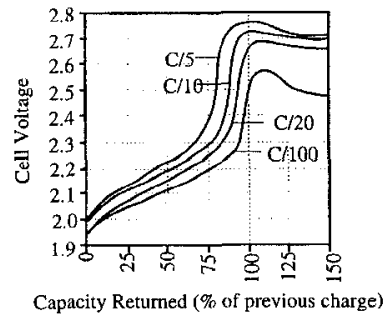

Fig. 1. Cell voltage versus capacity returned as percent of previous charge.

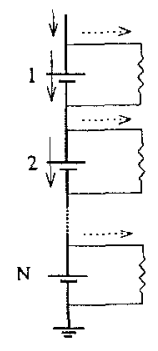

Fig. 2. Dissipative resistive shunts.

pulse of much shorter duration is applied to position the electrolyte ions away from the plates. Finally, a stabilization/rest period is used to position the ions at an optimum distance from the plate surfaces. Battery cell monitoring is used to optimize the charging algorithm.

Other schemes and algorithms have been reported in the literature as well. However, since all of these schemes deal with the battery pack as a whole, individual cell equalization cannot be easily achieved. Normal differences in cell chemistries and temperature gradients along a battery stack can lead to large nonuniformities in cell charge levels and corresponding cell terminal voltages. As a result, some of the modules will charge quicker than others. Continued high rate charging may well result in overcharging these modules. On the other hand, if the charging process is stopped when some of the modules are fully charged, then the undercharged modules can go into polarity reversal during deep discharge. The capacity of the battery in this case will not be fully utilized.

In order to prevent the adverse effects of unequalized charging of battery modules, individual modules need to be maintained at an equalized charge level. The property of individual cell voltages having the same value once they have reached the final state of charge can be utilized to achieve this task.

A simple approach to equalize the battery cells in a stack is to use bypass resistive shunts across each cell/module as shown in Fig. 2. The amount of current drawn by the shunt elements is proportional to the cell voltage which results in more current being diverted to the shunt as the cell voltage increases. This tends to reduce the voltage differences between the different cells within the stack since higher voltage 


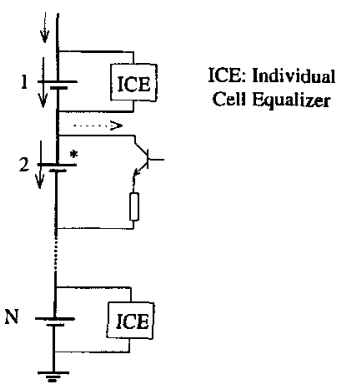

Fig. 3. ICEs.

cells are further discharged by the shunt elements. One drawback of this approach is that the recovered energy is being converted into additional losses in the shunt elements. In addition, the amount of current drawn by the shunt elements is not regulated. As a result, the cell voltages are not fully regulated.

In order to regulate the current drawn by the shunt elements, active circuitry can be used. The individual cell equalizers (ICE) shown in Fig. 3 are one such approach [5-7]. The ICE is a voltage-controlled current shunt which diverts the current away from the cell during trickle charging. This scheme prevents fully charged cells from getting overcharged while undercharged modules can still be trickle charged. In this scheme, the amount of lost energy is minimized since the shunt circuitry is only active when the cell voltage exceeds the preset level.

The ICE scheme is best suited for low charge/discharge current levels $(0.1 \% \ldots 1 \%)$. In these applications, the active devices can be signal level devices where the maximum shunt current is in the order of few hundred milliamps. This will also limit the energy dissipated in the shunt resistors.

Other schemes for charge equalization of series connected battery strings have been proposed in the literature [8-11]. These schemes were based on having a dedicated dc-to-dc converter stage across each battery module or a single equalization converter with a multiwinding transformer. Having a dedicated dc-to-dc converter stage for each battery module is considered expensive due to the extra hardware and software required. On the other hand, a single converter stage with a multiwinding transformer is more attractive. However, the nonideal characteristics the multiwinding transformer can greatly impact the performance of these equalization schemes.

\section{PROPOSED CHARGE EQUALIZATION SCHEME}

A new scheme for charge equalization of a series connected battery string has been proposed by the authors in [1]. The new proposed scheme consists of two parts: a bulk/fast charging system and a charge equalization system, as shown in Fig. 4. During fast/bulk charging, each individual battery module, or a stack of modules, is monitored for overcharging

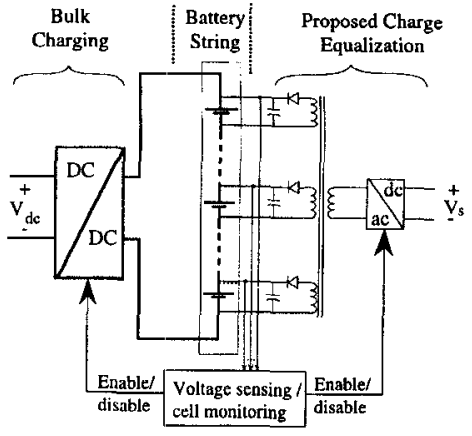

Fig. 4. Block diagram of proposed charging system.

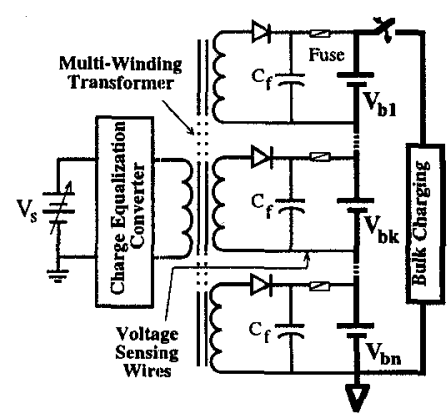

Fig. 5. Charge equalization of $n$-battery cells.

via the voltage sensing wires. If any of these stacks reaches its nominal voltage, or if gassing is detected, bulk charging is shut off and charge equalization is enabled. The sensing wires are also used by the charge equalization circuitry.

The charge equalization technique utilizes a simple isolated dc-to-dc converter with a capacitive output filter along with a multiwinding transformer as shown in Fig. 5. The coaxial transformer is known for its properties of low and controlled leakage inductance and accurate control of all fluxes $[12,13]$. These properties form the basis for realizing a fully symmetrical circuit.

The transformer and converter design need to be optimized so that the full converter rating can be used to charge the weakest module (stack of modules), and will gradually phase back into equal charging currents for all modules as the individual module voltages equalize. Once the final equalization voltage is reached, the converter can be turned off, if so desired. The charge equalizer can be operated in parallel with the bulk charger to optimally charge the battery stack.

\section{MULTIWINDING TRANSFORMERS}

As stated earlier, the proposed scheme utilizes a multiwinding transformer to equalize individual modules independently. The main challenge is to realize a transformer structure with controlled parasitics and highly symmetrical transfer characteristics. 


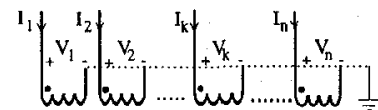

Fig. 6. Circuit diagram of $n$-circuit transformer.

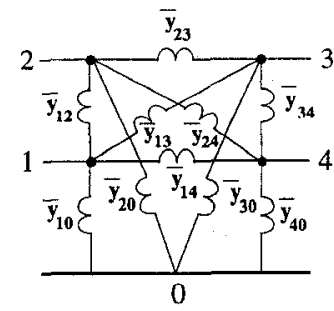

(a)

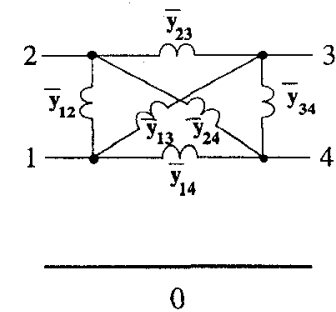

(b)
Fig. 7. Equivalent circuit of 4-circuit transformer.

\section{A. Multicircuit Transformer Theory}

Multicircuit transformer theory has been discussed in detail in many references $[14,15]$. Fig. 6 shows a transformer having $n$ windings all placed on a common core. In this analysis, the currents taken by the transformer capacitances are negligibly small except at very high frequencies and hence are neglected. As a result, the broken-line ground connection assumed in Fig. 6 will not alter the relations among the terminal voltages of the various windings.

The $n$-circuit transformer can be analyzed using node-current equations with each node magnetically coupled to all other nodes. The steady state vector current equations can be written as

$$
\mathbf{I}=\mathbf{Y} \cdot \mathbf{V}
$$

where $I$ is the vector of winding currents, $V$ is the vector terminal voltage of the windings, and $\mathbf{Y}$ is the complex self- and mutual admittances of the nodes. In (1), $Y_{i i}$ is the self admittance of node $i$ while $Y_{i j}$ is the mutual admittance of nodes $i$ and $j$.

In order to represent an $n$-circuit transformer with an equivalent circuit, the equivalent circuit must have the same number of free terminals, i.e., $2 n$ terminals. However, if the currents taken by transformer capacitances are neglected, an $n$-circuit transformer can be represented by an equivalent circuit with $n+1$ terminals. Fig. 7 (a) shows an equivalent circuit of a 4-circuit transformer. The equivalent circuit of Fig. 7(a) can be further simplified if the magnetizing currents are very small compared with the winding currents. In this case, the excitation admittances, $\bar{y}_{i 0}$ can be omitted. The equivalent circuit reduces to that shown in Fig. 7(b).

The values of the branch admittances of Fig. 7 can be determined experimentally by a number of tests where one of the windings is excited with all others short circuited.

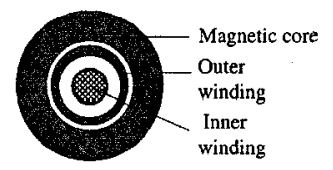

Fig. 8. CWT cross section.

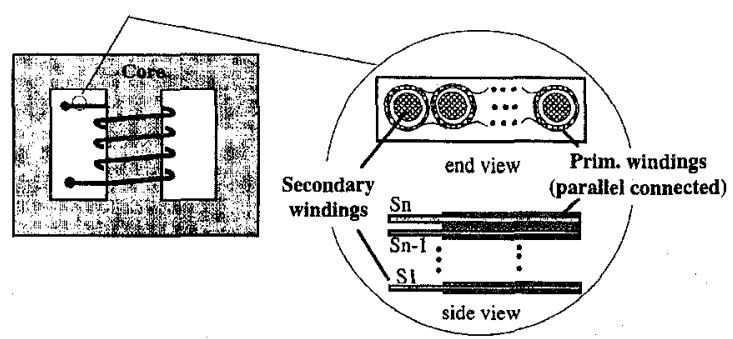

Fig. 9. Multiwinding CWT structure.

\section{B. Multiwinding Coaxial Winding Transformer}

As pointed out earlier, the challenge in implementing the proposed charge equalization technique lies in realizing a multiwinding transformer with symmetrical and tightly controlled parasitics. Yet, the transformer structure needs to be simple and inexpensive. The CWT has been demonstrated to have these properties $[12,13]$. A cross section of a $1: 1$ CWT is shown in Fig. 8.

Since the inner winding is totally enclosed by the outer winding, all the flux produced by the outer winding will link the inner one. In addition, the leakage field can only exist in the winding space between the inner and the outer windings. As a result, the leakage inductance can be controlled by controlling the interwinding space.

A multiwinding CWT structure for this implementation has been proposed in [1]. Fig. 9 shows a cross sectional view of the winding structure for a multiwinding CWT. As shown in Fig. 9, the transformer winding is a bundle of shielded or coaxial cables wound around a magnetic core. The cross section of the winding shows a bundle of three coaxial cables where the outer conductors, connected in parallel, form the primary winding while each of the inner conductors form one of the secondary windings.

\section{Equivalent Circuit of A Multioutput CWT}

The equivalent circuit of a multiwinding transformer has been shown earlier in Fig. 7 . However, for a multioutput CWT, the equivalent circuit can be further simplified. This is due to the fact that the leakage field between the primary winding and each of the secondary windings is dominated by the spacing between each individual secondary and its corresponding primary tube. At high frequencies, proximity effects force the bulk of the return current of each of the secondary windings to exist primarily in their corresponding outer primary tube. As a 


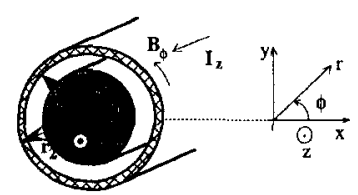

Fig. 10. Cross section of CWT winding.

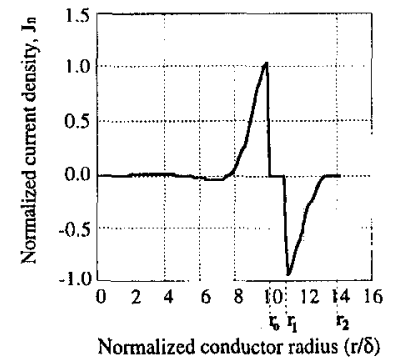

Fig. 11. Current density distribution of CWT winding.

result, very little leakage coupling exists between the individual secondary windings. This phenomenon can be seen by carrying out a field analysis for one of the windings as shown in Fig. 10.

The problem of Fig. 10 can be solved using Maxwell's equations in cylindrical coordinates. The resulting current density distribution within the inner and outer conductors are

$$
\begin{aligned}
& J_{z i}(r)=\frac{k I \cdot \mathbf{I}_{0}(k r)}{2 \pi r_{0} \cdot \mathbf{I}_{1}\left(k r_{0}\right)} \\
& J_{z 0}(r)=\frac{k I}{2 \pi r_{1}} \cdot \frac{\mathbf{K}_{1}\left(k r_{2}\right) \mathbf{I}_{0}(k r)+\mathbf{I}_{1}\left(k r_{2}\right) \mathbf{K}_{0}(k r)}{\mathbf{I}_{1}\left(k r_{1}\right) \mathbf{K}_{1}\left(k r_{2}\right)-\mathbf{I}_{1}\left(k r_{2}\right) \mathbf{K}_{1}\left(k r_{1}\right)}
\end{aligned}
$$

where $\mathbf{I}_{1}$ and $\mathbf{K}_{1}$ are the modified Bessel functions of the first and second kind of order one, while $k$ is defined as

$$
k=\frac{(1+j)}{\delta} ; \quad \delta=\sqrt{\frac{2}{\omega \mu \sigma}}
$$

where $\delta$ is the skin depth. The current density distribution within the winding is shown in Fig. 11. In this figure, the ac current density has been normalized to the value corresponding to a uniform current density distribution over one skin depth. In this case, the thickness of the outer shield is assumed to be $3 \delta$ while the inner winding radius is assumed to be $10 \delta$. These are typical dimensions at high frequencies.

As shown in Fig. 11, due to proximity effects, the bulk of current is concentrated near the winding space between the inner and outer windings which is the high field region. This justifies the assumption that at high frequencies, very little leakage coupling exists between the separate secondary windings. The equivalent circuit of a multioutput CWT is shown in Fig. 12 which is obtained by omitting the secondary-to-secondary coupling admittance links in Fig. 7(b).

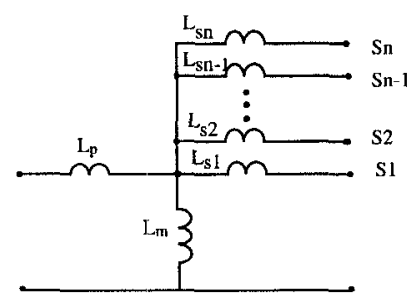

Fig. 12. Equivalent circuit of multioutput CWT.

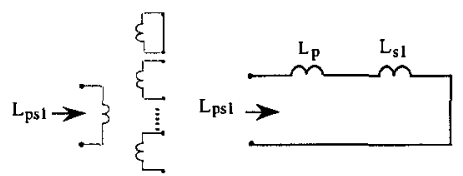

(a)

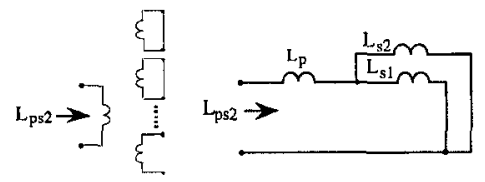

(b)

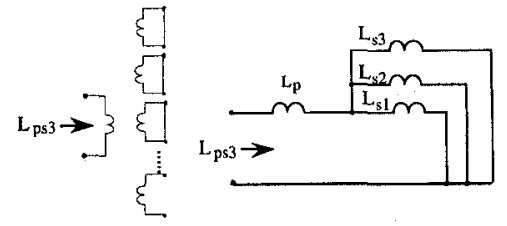

(c)

Fig. 13. Experimental verification of equivalent circuit.

\section{Experimental Verification of the Equivalent Circuit}

In order to validate the equivalent circuit of a multiwinding CWT that was proposed earlier in Fig. 12, experimental verification is used. This verification was done by performing a set of short circuit tests. The experimental setting consisted of using an HP4263 RLC meter and a 10-winding prototype transformer as shown earlier in Fig. 12. The measurements were done at $50 \mathrm{kHz}$. It was assumed that all secondary windings have the same secondary side leakage inductance. This assumption will be nearly true if the transformer windings are wound in a symmetrical fashion. With this assumption,

$$
\begin{aligned}
& L_{s i}=L_{s j}=L_{s} \quad i, j=1,2, \ldots 9, \quad i \neq j \\
& L_{m} \gg L_{s i} .
\end{aligned}
$$

The secondary terminal inductances are measured with one and two secondary windings short circuited separately as shown in Fig. 13(a) and 13(b), respectively. The leakage inductances $L_{p}$ and $L_{s}$ can be calculated with the proposed equivalent. To cross check the calculated $L_{p}$ and $L_{s}$, one of the remaining secondary windings is short circuited to make a new set of data to calculate $L_{p}$ and $L_{s}$ again as shown in 
Fig. 13(c). If the proposed equivalent circuit is correct, the results will be consistent.

For the ten-winding multiwinding CWT, the results with one and two secondary windings short circuited were

$$
L_{p s(1)}=0.19 \mathrm{mH} ; \quad L_{p s(2)}=0.098 \mathrm{mH}
$$

where

$$
\begin{aligned}
& L_{p s(1)}=L_{p}+L_{s}=0.19 \mathrm{mH} \\
& L_{p s(2)}=L_{p}+\frac{1}{2} \cdot L_{s}=0.098 \mathrm{mH} .
\end{aligned}
$$

Solving (8) and (9) simultaneously yields

$$
\left(L_{p}, L_{s}\right)=(0.006,0.184) \mathrm{mH} .
$$

Now, with a third winding short circuited, the measured inductance was

$$
L_{p s(3)}=0.067 \mathrm{mH}
$$

where

$$
L_{p s(3)}=L_{p}+\frac{1}{3} \cdot L_{s}=0.067 \mathrm{mH} .
$$

The inductances can be also calculated by solving (12) with either (8) or (9). Solving (8) and (12) simultaneously gives

$$
\left(L_{p}, L_{s}\right)=(0.0059,0.184) \mathrm{mH} \text {. }
$$

It is clear from (10) and (13) that the results are identical. The preceding experiment was carried out for all winding combinations and the results were totally consistent.

\section{IMPLEMENTATION AND DESIGN CONSIDERATIONS}

The proposed charge equalization scheme consists of a dc-to-dc converter, a multiwinding transformer and capacitive output filters. The implementation of the proposed scheme, as applied to an EV battery system, and the ratings of the different components are discussed in this section.

\section{A. System Level Design}

A battery charging system has three fundamental modes to fully charge a battery stack: bulk charging, trickle charging, and charge equalization. The trickle charge function can be performed by either stage of the proposed charging scheme (Fig. 4). If the trickle charge function is assumed by the bulk charger, the charge equalizer can be directly fed off the battery so as to redistribute charge within the stack (Fig. 14a). However, the bulk charger is normally not optimized for trickle charge operation. On the other hand, if the charge equalizer of Fig. 4 is operated after the termination of bulk charging, it must assume both the charge equalization function as well as the trickle charge function. This is rather a more optimal

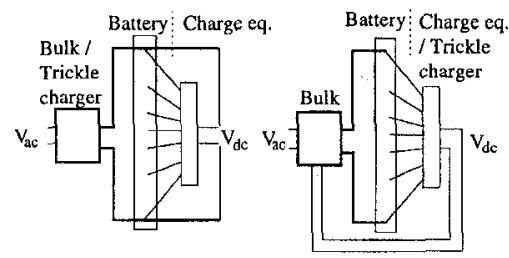

(a)

(b)

Fig. 14. System level implementation with trickle charge.

solution since the charge equalizer is optimized for low power operation. In this case, both the bulk charger and the charge equalizer will be fed from the same ac line (Fig. 14b).

The charge equalization converter can be operated simultaneously with bulk charging to optimally charge the battery stack to reduce the amount of time it takes to equalize a battery stack. These different modes of operation impact the charge equalizer power ratings.

\section{B. Converter Ratings}

The power ratings of the charge equalization converter depend mainly on the battery rating and specifications in addition to the battery state of charge after high rate charging is terminated.

As an example, the battery capacity of the GM Impact $\mathrm{EV}$ is in the range of $13 \mathrm{KWh}$. Assuming that the battery state of charge after high rate charging is $90 \%$, and since the charge equalizer assumes both trickle and charge equalization functions, the charge equalization stage has to supply the additional $10 \%$. As a result, the charge equalization converter rating will be $1300 \mathrm{Wh}$. If charge equalization is set to take about $4 \mathrm{~h}$, a $300 \mathrm{~W}$ converter will be needed.

In the above example, if the battery state of charge after high rate charging is less than $90 \%$, a higher converter rating will be needed. Alternatively, the same proposed converter can be used if the charge equalizer/trickle charger is allowed to take a longer time. In addition, the converter rating can be further lowered if charge equalization is enabled before the termination of bulk charging. This will preferentially pump more power into the weakest modules resulting in a higher battery state of charge when bulk charging is terminated.

\section{Charging Algorithm}

The charge equalization function is to direct charge from a source to the weakest modules in the battery stack. The weakest modules tend to have lower voltages relative to the fully charged ones, assuming the temperature and age of all the modules are the same.

The proposed charging algorithm is based on first raising the source voltage to a level which is higher than the lowest voltage module within the 


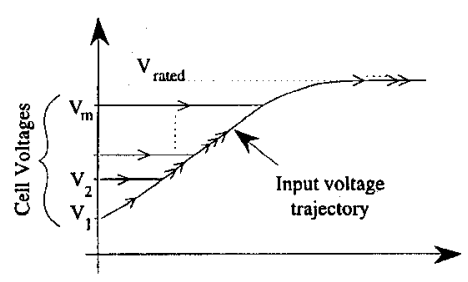

Fig. 15. Input voltage control for charge tapering.

battery stack but lower than the second lowest voltage module. For example, for a nominal module voltage of $13.4 \mathrm{~V}$, if the lowest voltage module is $12 \mathrm{~V}$ and the second lowest voltage module is $12.5 \mathrm{~V}$, the dc source voltage is raised to be greater than 12 but less than $12.5 \mathrm{~V}$. This will guarantee that the charging current will be diverted to the lowest module within the stack, i.e., the $12 \mathrm{~V}$ module. Next when the lowest module voltage reaches the second lowest module voltage, the dc source voltage is raised to be higher than the second lowest module voltage but lower than the third lowest module voltage, thus ensuring that the charging current is equally divided between the lowest two modules within the stack. As the lowest two module voltages reach the third lowest module voltage, the source voltage is raised again in a similar fashion. The process continues until all the stack is charged. This guarantees optimal charging for the battery stack. This proposed charging algorithm is shown in Fig. 15.

The above algorithm is based on the fact that the secondary-to-secondary leakage coupling is minimal and each secondary circuit is identical. As a result, the equivalent circuit of a multiwinding CWT degenerates into a leakage inductance between the single primary winding and each of the secondary windings with no secondary-to-secondary leakage component as shown earlier in Fig. 12. In addition, all leakage inductances are the same. The per-winding equivalent circuit is shown in Fig. 16. With a forward type converter topology, applying voltage to the primary winding will result in a current flowing in each secondary winding which only depends on the difference between the supply voltage $V_{s}$, the battery module voltage, $V_{b}$, and the leakage inductance $L_{l k}$ as given by

$$
I_{c h}(t)=\frac{V_{s}-V_{b}}{L_{l k}} t .
$$

It is clear from the above equation that if $V_{s}$ is less than $V_{b}$, the current would be negative. However, since the secondary rectifier diode can only allow positive current flow, the diode will not conduct and the secondary current will be zero. When the source voltage level is raised to a level which is higher than the lowest voltage module within the battery stack but lower than the second lowest voltage module, the only non-zero charging current will flow through the lowest voltage module while all other currents will be zero.

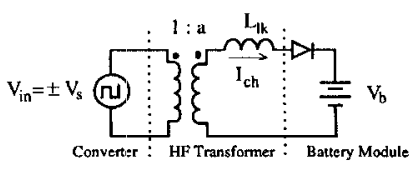

Fig. 16. Per-winding equivalent circuit for capacitive output forward converter.

\section{Converter Topologies}

The specific choice of a multiple output $\mathrm{dc} / \mathrm{dc}$ converter topology impacts the simplicity and control of the charge equalization scheme. The charge equalization function is to direct charge from a source to the weakest modules in the battery stack. The weakest modules tend to have lower voltages relative to the fully charged ones, assuming the temperature and age of all the modules are the same. Two simple topologies which transfer current (charge) to the lowest voltage outputs are the capacitive output forward converter and the flyback converter.

The application of the capacitive output forward converter for charge equalization using the CWT was shown in [1]. With forward converters, energy is delivered to the load when the switch is conducting. This allows optimal utilization of the charge equalization algorithm discussed in the previous section.

The transformer and converter need to be designed so that the full converter rating can be used to charge the weakest module and gradually phase back into equal charging currents for all modules as their voltages equalize. By using a preregulator stage, the input voltage can be controlled to coincide with the voltage of the weakest module within the stack and then increased slowly to charge other modules as shown earlier in Fig. 15. In this figure, the ascending order subscripts refer to the order of the weakest modules.

Another possible topology for charge equalization is the flyback converter. The flyback converter ideally transfers a specific amount of energy to the lowest voltage module every switching cycle. A simple input voltage feed-forward control scheme is needed to regulate the input energy per cycle. The transfer of energy to the lowest voltage module is accomplished via a well coupled multiwinding transformer. If the secondary-to-secondary leakage coupling is weak, the stored energy will be distributed over many modules, especially the weakest ones. However, optimal charging is not achieved since the charging current cannot be directed solely to the weakest cell within the stack but rather distributed among a combination of cells.

\section{E. Transformer Design Considerations}

As pointed out earlier, the implementation of the proposed charge equalization scheme requires 


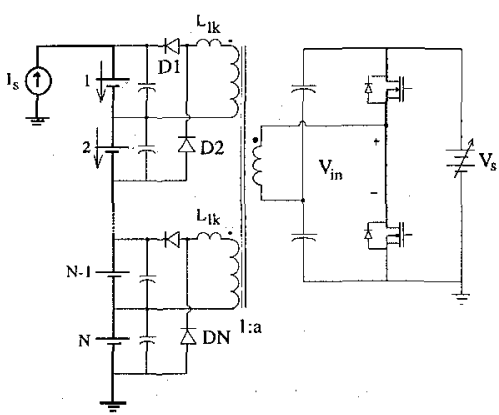

Fig. 17. Reducing number of secondary windings for centralized forward converter scheme.

transformers with symmetrical characteristics, low and controlled leakage inductances with multiple outputs to achieve a quick and well balanced equalization scheme. The coaxial wound transformer was shown to be an excellent choice for the capacitive output forward converter scheme. A conventional foil wound transformer can also be used but the relative equalization rate between modules will no longer be linear with module voltage.

To take advantage of the CWTs characteristics, a $1: 1$ turns ratio results between each of the primary and secondary windings (Fig. 9). This would require a low voltage source which is approximately equal to the module rated voltage. Since the charge equalizer is supplied by a high voltage dc source (Fig. 14), a step down stage is needed. In addition, in order to provide control to direct charge to the weakest module within the stack, an additional preregulator stage is required. These two stages can be either combined or a step down transformer with a simple preregulator can be used.

In an EV application, the number of battery modules is relatively high, namely 20 to 30 series connected modules. A CWT design with 20 to 30 secondaries may become quite cumbersome or impractical. One way to reduce the number of secondary windings is to combine each pair of modules and treat them as a single module. However, individual modules within the pairs are not equalized.

In order to reduce the number of secondary windings, a half-bridge dc-to-dc converter can be employed where bi-directional power flow is utilized. Due to the bidirectional excitation, the number of secondary windings can be cut in half as shown in Fig. 17. During the positive cycle, the rectifying diode D1 will be on which results in charge transfer into cell 1 . During the negative cycle, the rectifying diode D2 will be on resulting in charge transfer into cell 2. Filter capacitors are utilized across each battery module to filter out the high frequency charging currents. Other types of bidirectional power converters can be used as well.

Using the above-mentioned approach, for an EV application of 20 to 30 series connected battery

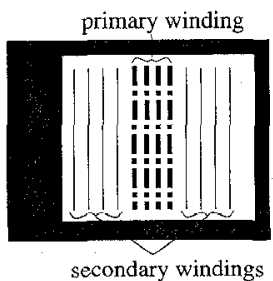

Fig. 18. Example for $(4: 1: 1: 1: 1)$ winding structure.

modules, a 10 to 15 winding transformer can be used which is more practical.

Unlike the forward topology, the flyback topology, does not benefit significantly from the CWT technology. This is due to the fact that the flyback topology transfers stored energy in the magnetizing inductance of the transformer, rather than the primary side, to the secondary side. With a flyback converter topology, a conventional foil wound transformer with step down turns ratio is used where a high voltage bus can be used to feed the converter. In this case, attention must be paid to minimize the leakage inductances between secondaries. An example of a $(4: 1: 1: 1)$ winding structure for a flyback implementation is shown in Fig. 18.

\section{F. Capacitive Filter Ratings}

The capacitive output filters are needed to filter out the high frequency ripple supplied by the converter in addition to decoupling the unequal lead inductances between the transformer secondaries and the individual battery modules.

The rating of the capacitive filter elements depends on the converter topology and rating. Since both the forward and flyback topologies have triangular current waveforms, the rms capacitor current is nearly the same as the average output current. In addition, the voltage rating of these capacitors is the rated module voltage.

For the $300 \mathrm{~W}$ converter rating proposed earlier, and assuming a maximum average winding current of $6 \mathrm{~A}$, a $15 \mathrm{~V} / 6 \mathrm{~A}$ capacitor is needed. The value of capacitance is a function of the switching frequency and the lead inductance between the transformer secondaries and the individual battery modules. The minimum capacitance needed is given by,

$$
C_{f} \gg \frac{1}{4 \pi^{2} f_{s}^{2} L_{f}}
$$

where $f_{s}$ is the switching frequency and $L_{f}$ is the total secondary filter inductance. At $100 \mathrm{kHz}$, and assuming a minimum of $1.0 \mu \mathrm{H}$ of lead inductance, a minimum of a $2.5 \mu \mathrm{F}$ capacitor is needed.

\section{G. Protection}

The most serious failure mode during charge equalization is having a low voltage cell or a dead 
cell within a module. As a result, the charging current of this module may be quite high. Such a module would continue accepting a high rate charging current without affecting its terminal voltage. A low voltage module can be detected using the voltage sensing wires across it. This can be achieved by comparing the measured voltage with a preset lower threshold below which a control action is taken. If this condition is detected, it is desirable to disconnect the low voltage module while continuing to provide equalization for the rest of the stack.

A simple protection scheme for the above mentioned failure mode can be implemented using fuses in series with each of the secondary windings as shown earlier in Fig. 5. If a module voltage falls below a certain threshold, the controller action will be such that the fuse of its corresponding winding is cleared. This can be achieved by raising the input voltage so as to cause a current higher than the fuse rating. Once the fuse clears, the failed module is disconnected from the equalizer and charge equalization can proceed for the rest of the stack. The main control unit would then inform the user about the faulty modules so they can be repaired or replaced.

\section{EXPERIMENTAL VERIFICATION}

In order to verify the concepts presented in this work, two $100 \mathrm{~W}$ prototype charge equalization converters using the forward and flyback topologies, were built in the lab.

For the forward converter implementation, a three winding CWT was built in the lab. The operating frequency was selected to be $50 \mathrm{kHz}$. The resultant leakage and magnetizing inductances were measured to be:

$$
\begin{array}{ll}
L_{m}=170 \mu \mathrm{H} ; & L_{s 1}=320 \mathrm{nH} \\
L_{s 2}=320 \mathrm{nH} ; & L_{s 3}=310 \mathrm{nH} .
\end{array}
$$

Three Titan $12 \mathrm{~V}$ deep cycle gel-cell batteries from Exide were used in series. Their full charge voltage was $13.6 \mathrm{~V}$ and they can be discharged down to $9.5 \mathrm{~V}$. These modules were charged/discharged individually to have different initial state of charge. The initial value of the battery voltages were $12.37,13.07$, and 13.39 , from top to bottom, respectively. The charge equalization converter was enabled for $3.5 \mathrm{~h}$ and the individual cell voltages were recorded every $6 \mathrm{~min}$. The resulting cell voltages were plotted as shown in Fig. 19. It is clear from this figure that the individual cells charge to the same nominal value at the end of the charge equalization mode.

The secondary current wave forms at the beginning and end of charge equalization are shown in Fig. 20(a) and (b), respectively. Note here that the

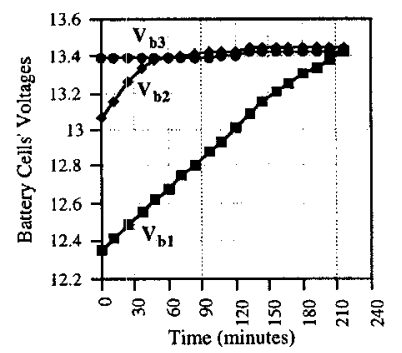

Fig. 19. Recorded module voltages with forward topology.

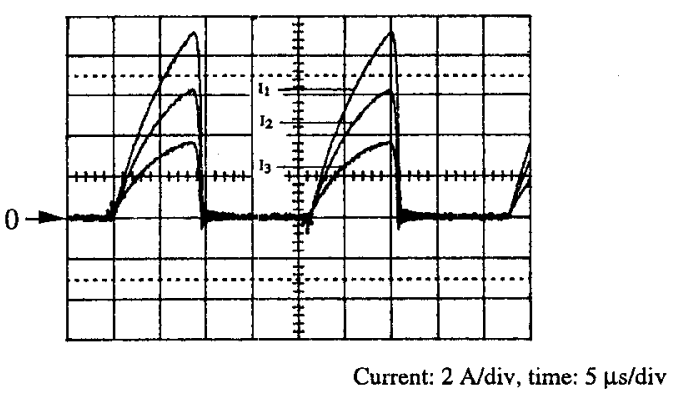

(a)

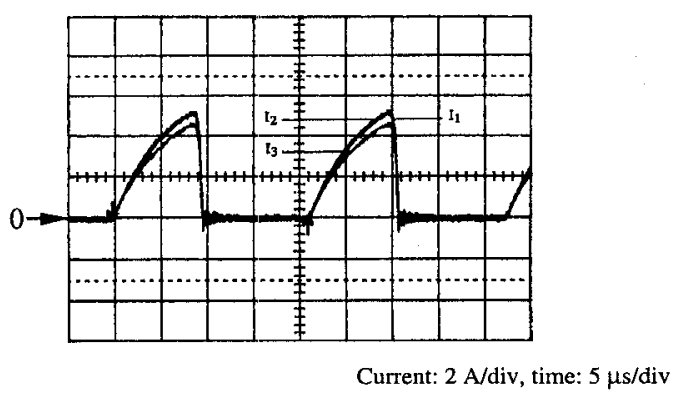

(b)

Fig. 20. Forward converter. (a) Secondary charging currents at $t=0$. (b) Secondary charging currents at $t=3.5$ hrs.

weakest cell draws more current than the other cells. As that cell charges, the charging current decreases as shown in Fig. 20(b).

For the flyback implementation, a $3: 1: 1: 1$ conventional foil wound transformer was built. The switching frequency was chosen to be $50 \mathrm{kHz}$ and the duty cycle of the transistor was set to $45 \%$ to guarantee the reset of the magnetizing energy. The same battery modules were used with the same initial state of charge. The charge equalization converter was enabled for $2 \mathrm{~h}$ and the individual cell voltages were recorded every $6 \mathrm{~min}$. The resulting cell voltages were plotted as shown in Fig. 21.

The secondary current wave forms at the beginning and end of charge equalization are shown in Fig. 22(a) and (b), respectively. As in the previous case, the individual cells charge to the same nominal value at the end of the charge equalization mode. However, with the flyback implementation, the charge cannot be directed to the weakest cell due to the secondary-to-secondary coupling. 


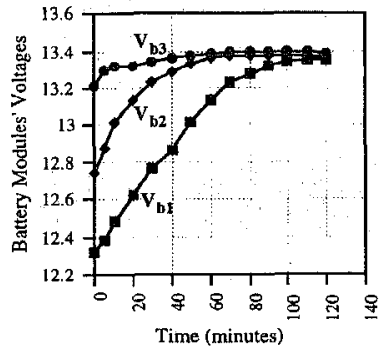

Fig. 21. Recorded module voltages with flyback topology.

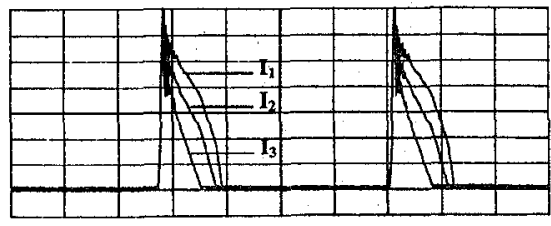

Current: $2 \mathrm{~A} / \mathrm{div}$, time: $5 \mu \mathrm{s} / \mathrm{div}$

(a)

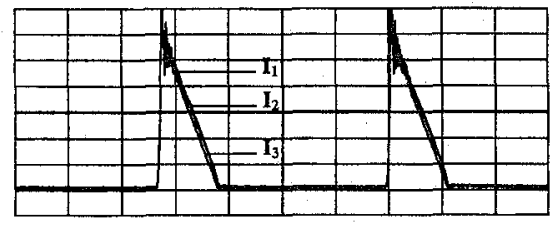

Current: 2 A/div, time: $5 \mu \mathrm{s} / \mathrm{div}$

(b)

Fig. 22. Flyback converter. (a) Secondary charging currents at $t=0$. (b) Secondary charging currents at $t=3.5 \mathrm{hrs}$.

\section{CONCLUSIONS}

In this paper, design considerations for charge equalization of an EV battery system were discussed. The proposed scheme utilizes a simple isolated dc-to-dc converter with a multiwinding transformer. The main features of the proposed scheme include:

1) a simple converter topology serving both trickle charging and charge equalization functions,

2) utilizes transformers with highly symmetrical transfer characteristics (CWT),

3 ) utilizes the sensing wires for monitoring and charge equalization,

4) provides a low cost implementation,

5) provides improved battery stack life.

\section{REFERENCES}

[1] Kutkut, N. H., Divan, D. M., and Novotny, D. W. (1994) Charge equalization for series connected battery strings. In Proceedings of the IEEE IAS Annual Meeting, Oct. 1994, 1008-1015.

[2] Unitrode Corp. (1994) Unitrode Product and Applications Handbook, 1993-1994. Unitrode Corporation, 1994.

[3] Chan, C. C., Leung, W. S., and Chu, K. C. (1990) A microprocessor based intelligent charger for electric vehicle lead acid batteries. In Proceedings of the Electric Vehicle Symposium, EVS-10, Hong Kong, (1990), 456-466.
[4] Podrazhansky, Y., and Popp, P. W. (1989)

Rapid battery charger, discharger and conditioner. U.S. Patent 4,829,225, May 9, 1989.

[5] Bjork, D. (1986)

Maintenance of batteries-New trends in batteries and automatic battery charging.

In INTELEC Conference Proceedings, 1986, 355-360.

[6] Bergvik, S., and Bjorkstrom, L. (1984)

Prolonged useful life and reduced maintenance of lead-acid batteries by means of individual cell voltage regulation.

In INTELEC Conference Proceedings, 1984, 63-66.

[7] Lindemark, B. (1991)

Individual cell voltage equalizers (ICE) for reliable battery performance.

In INTELEC Conference Record, Kyoto, Japan, 1991, 196-201.

[8] Cox, J. (1978)

Charging system and method for multicell storage batteries.

U.S. Patent 4,079,303, Mar. 14, 1978.

[9] Davis, J. (1991)

Battery charger for charging a plurality of batteries.

U.S. Patent 5,003,244, Mar. 26, 1991.

[10] Schmidt, H., and Siedle, C. (1993)

The charge equalizer-A new system to extend battery lifetime in photovoltaic systems, U.P.S. and electric vehicles.

In INTELEC Conference Proceedings, 1993, 146-151.

[11] Tung, S. T., Hopkins, D. C., and Mosling, C. R. (1993) Extension of battery life via charge equalization. IEEE Transactions on Industrial Electronics, 40 (Feb. 1993), 96-104.

[12] Rauls, M. S., Novotny, D. W., and Divan, D. M. (1993) Design considerations for high frequency coaxial winding transformers.

IEEE IAS Transactions, 29, 2 (Mar./Apr. 1993), 375-381.

[13] Rauls, M. S., Novotny, D. W., Divan, D. M., Bacon, R., and Gascoigne, R. W. (1992)

Multi-turn high frequency coaxial winding power transformers.

In 1992 IEEE-IAS Annual Meeting Conference Record, Oct. 1992, 375-381.

[14] Blume, L. F. (1938)

Transformer Engineering.

New York: Wiley, 1938.

[15] M.I.T. staff (1943)

Magnetic Circuits and Transformers.

New York: Wiley, 1943.

[16] Kutkut, N. H., Wiegman, H. L. N., Divan, D. M., and Novotny, D. W. (1995)

Design considerations for charge equalization of an electric vehicle battery system.

In IEEE APEC Conference Record, 1995, 96-103.

[17] Hubert, T. (1995)

A battery system using adaptive run-time estimation software controlled multi-mode charging and intrinsic diagnostics, contribute to enhance UPS reliability. In HFPC Conference Record, 1995, 382-395.

[18] Linden, D. (1995) Handbook of Batteries. New York: McGraw-Hill, 1995.

[19] Berndt, D. (1994) Maintenance Free Batteries. New York: Wiley, 1994.

[20] Reid, D. P., and Glasa, I. (1984)

A new concept: Intermittent charging of lead acid batteries in telecommunications systems. IEEE 1984, Ch 2073-5/84/0000-067. 


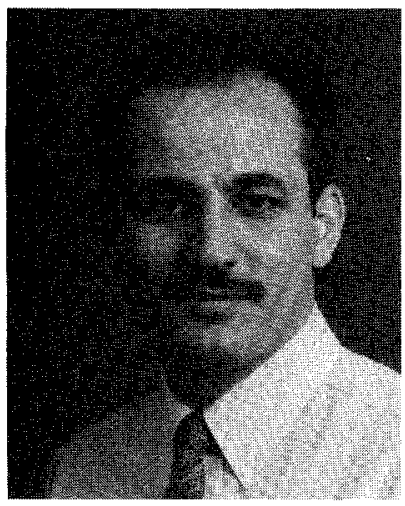

Nasser H. Kutkut Nasser Kutkut (M'89) received his B.Sc. degree in electrical engineering from Jordan University of Science and Technology, Irbid, Jordan, in 1989, the M.Sc. in electrical engineering and computer science from the University of Illinois at Chicago in 1990, and the Ph.D. degree in electrical engineering from the University of Wisconsin-Madison in 1995.

He joined Soft Switching Technologies of Middleton, WI in 1995 as a Senior Design Engineer where he is involved in the design and development of power electronic apparatus and systems. His main research interests include soft switching converter topologies, high power converters, high frequency magnetics design, in addition to electric vehicle battery charging and equalization.

Dr. Kutkut is a member of Sigma Xi, New York Academy of Science, the American Association for the Advancement of Science (AAAS) and the IEEE PELS and IA Societies. He won the second prize paper award of the IEEE-IAS Industrial Power Converter Committee at the IAS-1994 annual meeting.

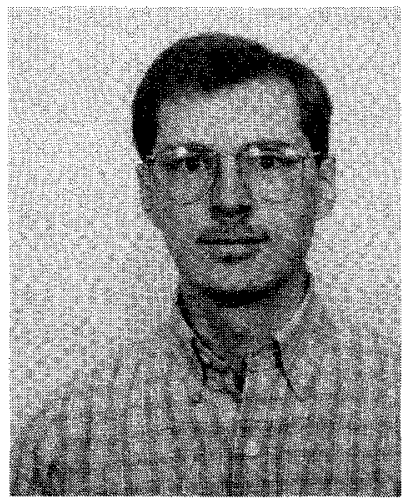

Herman Wiegman was born in New Hampshire in 1966, and he completed his undergraduate engineering degree at Worcester Polytechnic Institute in 1988. He then obtained an M.S. in power electronics and electric machines at the University of Wisconsin-Madison in 1991.

He joined General Electric's Corporate Research and Development Center in Schenectady, NY, in 1991, where he was responsible for developing custom power supply solutions and transitioning them to the GE businesses and other international firms. In 1994 he returned to UW-Madison to pursue a Ph.D. Since then his interests have moved towards developing high fuel economy, low emmission hybrid electric vehicles (HEVs). He and the student-run HEV team have had great success developing both series and parallel hybrid vehicles. His Ph.D. research focuses on applying an on-line electro-thermal model to predict the bidirectional power capability of power buffering batteries.

In 1996, Mr. Wiegman received a Netherlands--America Foundation fellowship, administered in close association with the Fulbright Commitee. He and his family are spending a year at the Technical University of Eindhoven, The Netherlands.

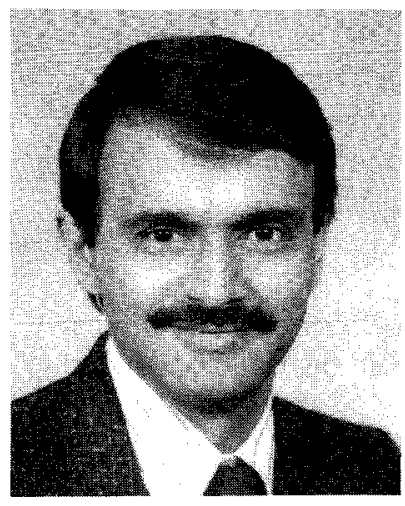

Deepak Divan (S'78-M'83-SM'91) obtained his B.Tech in electrical engineering from the Indian Instiute of Technology in 1975, and his M.S. and $\mathrm{Ph} . \mathrm{D}$. degrees in the same discipline from the University of Calgary, Canada, in 1979 and 1983, respectively.

He has been a Professor at the University of Wisconsin-Madison, since 1985, and is an Associate Director of the Wisconsin Electric Machines and Power Electronics Consortium (WEMPEC). He is presently on leave from the university and is President and CEO of Soft Switching Technologies Corporation, a manufacturer of power conversion equipment based in Middleton, WI.

Dr. Divan has 20 issued and pending patents, and over 90 technical publications including over 10 prize papers and a paper in the IEEE Spectrum. 


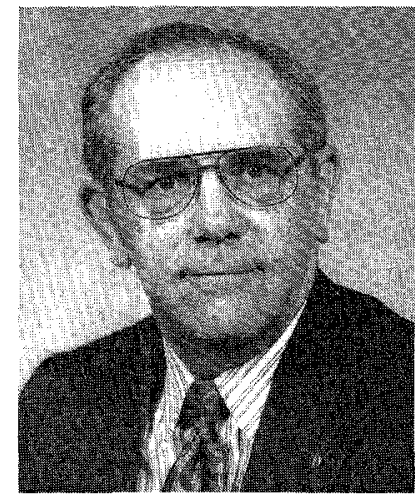

Donald W. Novotny (F'87) received his B.S. and M.S. degrees in electrical engineering from the Illinois Institute of Technology, Chicago, in 1956 and 1957 and the Ph.D. degree from the University of Wisconsin-Madison in 1961.

Since 1961 he has been a member of the faculty at the University of Wisconsin-Madison where he is currently Grainger Professor of Power Electronics and Co-director of the Wisconsin Electric Machines and Power Electronics Consortium (WEMPEC), an educational and research support organization with over 50 industry sponsors. He served as Chairman of the Electrical and Computer Engineering Department from 1976 to 1980 and as an Associate Director of the University-Industry Research Program from 1972 to 1974 and from 1980 to 1993 . He has been an active consultant to many organizations and has also been a Visiting Professor at Montana State University, the Technical University of Eindhoven, Eindhoven, Netherlands, the Catholic University of Leuven, Leuven, Belgium and a Fulbright Lecturer at the University of Ghent, Ghent, Belgium.

Dr. Novotny has published over 100 technical articles on electric machines, variable frequency drives and power electronic control of industrial systems, nine of which have received prize paper awards from the IEEE Industry Applications Society. He is a member of IEEE, ASEE, Sigma Xi, Eta Kappa Nu, and Tau Beta $\mathrm{Pi}$ and is a registered Professional Engineer in Wisconsin. 